The Journal of $\mathbf{N}_{\text {onlinear }} \mathbf{S}_{\text {cience and }}$ Applications

http://www.tjnsa.com

\title{
LOCAL CONVERGENCE ANALYSIS OF INEXACT NEWTON-LIKE METHODS
}

\author{
IOANNIS K. ARGYROS ${ }^{1 *}$ AND SAÏD HILOUT ${ }^{2}$
}

\begin{abstract}
We provide a local convergence analysis of inexact Newton-like methods in a Banach space setting under flexible majorant conditions. By introducing center-Lipschitz-type condition, we provide (under the same computational cost) a convergence analysis with the following advantages over earlier work [9]: finer error bounds on the distances involved, and a larger radius of convergence.

Special cases and applications are also provided in this study.
\end{abstract}

\section{INTRODUCTION}

In this study we are concerned with the problem of approximating a locally unique solution $x^{\star}$ of equation

$$
F(x)=0,
$$

where $F$ is a Fréchet-differentiable operator defined on a convex subset $\mathcal{D}$ of a Banach space $\mathcal{X}$ with values in a Banach space $\mathcal{Y}$.

A large number of problems in applied mathematics and also in engineering are solved by finding the solutions of certain equations. For example, dynamic systems are mathematically modeled by difference or differential equations, and their solutions usually represent the states of the systems. For the sake of simplicity, assume that a time-invariant system is driven by the equation $\dot{x}=Q(x)$, for some suitable operator $Q$, where $x$ is the state. Then the equilibrium states are determined by solving equation (1.1). Similar equations are used in the case of

Date: Received: January 2009.

* Corresponding author.

2000 Mathematics Subject Classification. Primary 65H10, 65G99, 90C30, 49M15, 47J20.

Key words and phrases. Inexact Newton-like method, Banach space, Majorant conditions, Local convergence. 
discrete systems. The unknowns of engineering equations can be functions (difference, differential, and integral equations), vectors (systems of linear or nonlinear algebraic equations), or real or complex numbers (single algebraic equations with single unknowns). Except in special cases, the most commonly used solution methods are iterative--when starting from one or several initial approximations a sequence is constructed that converges to a solution of the equation. Iteration methods are also applied for solving optimization problems. In such cases, the iteration sequences converge to an optimal solution of the problem at hand. Since all of these methods have the same recursive structure, they can be introduced and discussed in a general framework.

We use the inexact Newton-like method (INLM):

$$
x_{n+1}=x_{n}+s_{n}, \quad \mathcal{B}\left(x_{n}\right) s_{n}=-F\left(x_{n}\right)+r_{n} \quad(n \geq 0), \quad\left(x_{0} \in \mathcal{D}\right)
$$

to generate a sequence $\left\{x_{n}\right\}(n \geq 0)$ approximating $x^{\star}$. Here, $\mathcal{B}(x) \in \mathcal{L}(\mathcal{X}, \mathcal{Y})$ the space of bounded linear operators from $\mathcal{X}$ into $\mathcal{Y}$, are approximations of the Fréchet-derivative $F^{\prime}(x)$; the residuals $r_{n}$ satisfy

$$
\left\|\mathcal{P}_{n} r_{n}\right\| \leq \theta_{n} \quad\left\|\mathcal{P}_{n} F\left(x_{n}\right)\right\| \quad(n \geq 0)
$$

for a suitable forcing sequence $\left\{\theta_{n}\right\}$, and some invertible sequence $\left\{\mathcal{P}_{n}\right\}$ of preconditioners for the linear equation in $(1.2)$.

If $\mathcal{P}_{n}=I$, and $\mathcal{B}_{n}=F^{\prime}\left(x_{n}\right)(n \geq 0)$, we obtain the inexact Newton method (INM). A survey of convergence results under various Lipschitz-type conditions for (INM) and (INLM) can be found in [4], [5] (see also [1]-[3], [7]-[15]).

In this study, we are motivated by the elegant work in [9], and optimization considerations. We introduce center-Lipschitz-type conditions (see (2.2)), and use it to find upper bounds on the distances $\left\|F^{\prime}(x)^{-1} F^{\prime}\left(x^{\star}\right)\right\|(x \in \mathcal{D})$. This approach leads (under the same computational cost) to a local convergence analysis, with the following advantages: finer estimates for the distances $\left\|x_{n}-x^{\star}\right\|$ $(n \geq 0)$, and a larger convergence radius.

Special cases and applications are also provided in this study.

\section{LOCAL CONVERGENCE ANALYSis OF (INLM)}

We provide the main local convergence theorem for (INLM):

Theorem 2.1. Let $F: \mathcal{D} \subseteq \mathcal{X} \longrightarrow \mathcal{Y}$ be a continuously differentiable operator. Let $x^{\star} \in \mathcal{D}, R>0$, and set

$$
\kappa=\sup \left\{t \in[0, R): U\left(x^{\star}, t\right)=\left\{x \in \mathcal{X}:\left\|x-x^{\star}\right\|<t\right\} \subseteq \mathcal{D}\right\} .
$$

Assume:

$F\left(x^{\star}\right)=0, \quad F^{\prime}\left(x^{\star}\right)^{-1} \in \mathcal{L}(\mathcal{Y}, \mathcal{X})$ 
there exist functions $f_{0}, f:[0, R) \longrightarrow(-\infty,+\infty)$ continuously differentiable, such that:

$$
\begin{gathered}
\left\|F^{\prime}\left(x^{\star}\right)^{-1}\left[F^{\prime}(x)-F^{\prime}\left(x^{\star}\right)\right]\right\| \leq f_{0}^{\prime}\left(\left\|x-x^{\star}\right\|\right)-f_{0}^{\prime}(0), \\
\left\|F^{\prime}\left(x^{\star}\right)^{-1}\left[F^{\prime}(x)-F^{\prime}\left(x^{\star}+\tau\left(x-x^{\star}\right)\right)\right]\right\| \leq f^{\prime}\left(\left\|x-x^{\star}\right\|\right)-f^{\prime}\left(\tau \quad\left\|x-x^{\star}\right\|\right),
\end{gathered}
$$

for all $x \in U\left(x^{\star}, \kappa\right)$, and $\tau \in[0,1]$;

$$
\begin{aligned}
& f(0)=f_{0}(0), \quad \text { and } \quad f^{\prime}(0)=f_{0}^{\prime}(0)=-1 ; \\
& f_{0}^{\prime}, f^{\prime} \text { are convex and strictly increasing; }
\end{aligned}
$$

Choose: $\theta \in[0,1), w_{1}, w_{2}$, with $w_{2} \in\left[0, w_{1}\right)$, such that:

$$
w_{1} \theta+w_{2}<1 \text {. }
$$

Set:

$$
\begin{gathered}
v=\sup \left\{t \in[0, R): f^{\prime}(t)<0\right\}, \\
\rho_{0}=\sup \left\{t \in[0, v): w_{1}(1+\theta)\left(\frac{f(t)}{t f_{0}^{\prime}(t)}-\frac{f^{\prime}(t)}{f_{0}^{\prime}(t)}\right)+w_{1} \theta+w_{2}<1\right\},
\end{gathered}
$$

and

$$
\sigma_{0}=\min \left\{\kappa, \rho_{0}\right\}
$$

$\mathcal{B}\left(x_{n}\right)$ are invertible approximations of $F^{\prime}\left(x_{n}\right)(n \geq 0)$, and

$$
\begin{gathered}
\left\|\mathcal{B}\left(x_{n}\right)^{-1} F^{\prime}\left(x_{n}\right)\right\| \leq w_{1}, \\
\left\|\mathcal{B}\left(x_{n}\right)^{-1} F^{\prime}\left(x_{n}\right)-I\right\| \leq w_{2} ;
\end{gathered}
$$

the residuals $r_{n}(n \geq 0)$ satisfy

$$
\left\|\mathcal{P}_{n} r_{n}\right\| \leq \theta_{n} \quad\left\|\mathcal{P}_{n} F\left(x_{n}\right)\right\|
$$

for some invertible matrix sequence $\left\{\mathcal{P}_{n}\right\}(n \geq 0)$ preconditioners for the linear system in (INLM), and a forcing sequence $\left\{\theta_{n}\right\}(n \geq 0)$ of non-negative numbers satisfying

$$
\theta_{n} \operatorname{cond}\left(\mathcal{P}_{n} F^{\prime}\left(x_{n}\right)\right) \leq \theta \quad(n \geq 0) .
$$

Then, sequence $\left\{x_{n}\right\}$ generated by (INLM) is well defined, remains in $U\left(x^{\star}, \sigma_{0}\right)$, for all $n \geq 0$, and converges to $x^{\star}$.

Moreover, the following estimates hold for all $n \geq 0$ :

where,

$$
\left\|x_{n+1}-x^{\star}\right\| \leq \alpha_{0} \quad\left\|x_{n}-x^{\star}\right\|
$$

$0 \leq \alpha_{0}=w_{1}(1+\theta)\left(\frac{f\left(\left\|x_{0}-x^{\star}\right\|\right)}{\left\|x_{0}-x^{\star}\right\| f_{0}^{\prime}\left(\left\|x_{0}-x^{\star}\right\|\right)}-\frac{f^{\prime}\left(\left\|x_{0}-x^{\star}\right\|\right)}{f_{0}^{\prime}\left(\left\|x_{0}-x^{\star}\right\|\right)}\right)+w_{1} \theta+w_{2}<1$. 
Proof. By the definition of $v, x \in U\left(x^{\star}, \sigma_{0}\right)$, hypotheses 2.5), we deduce:

$$
f_{0}^{\prime}\left(\left\|x-x^{\star}\right\|\right)<0 .
$$

Using (2.2), we obtain in turn:

$\left\|F^{\prime}\left(x^{\star}\right)^{-1}\left(F^{\prime}(x)-F^{\prime}\left(x^{\star}\right)\right)\right\| \leq f_{0}^{\prime}\left(\left\|x-x^{\star}\right\|\right)-f_{0}^{\prime}(0)<-f_{0}^{\prime}(0)=1 \quad($ by 2.4 $)$

It follows from (2.16), and the Banach lemma on invertible operators [4], [5], [12] that $F^{\prime}(x)^{-1} \in \mathcal{L}(\mathcal{Y}, \mathcal{X})$, and

$$
\begin{aligned}
\left\|F^{\prime}(x)^{-1} F^{\prime}\left(x^{\star}\right)\right\| & \leq\left(1-\left\|F^{\prime}\left(x^{\star}\right)^{-1}\left(F^{\prime}(x)-F^{\prime}\left(x^{\star}\right)\right)\right\|\right)^{-1} \\
& \leq\left(1-\left(f_{0}^{\prime}\left(\left\|x-x^{\star}\right\|\right)-f_{0}^{\prime}(0)\right)\right)^{-1}=\frac{1}{\left|f_{0}^{\prime}\left(\left\|x-x^{\star}\right\|\right)\right|}
\end{aligned}
$$

since $\sigma_{0} \leq v$.

It follows that

$$
x_{+}=x+s, \quad \mathcal{B}(x) s=-F(x)+r
$$

is well defined.

In view of (INLM), and (2.18), we obtain the identity:

$$
\begin{aligned}
x_{+}-x^{\star}= & \mathcal{B}(x)^{-1}\left(F\left(x^{\star}\right)-\left(F(x)+F^{\prime}(x)\left(x^{\star}-x\right)\right)\right)+ \\
& \left(\mathcal{B}(x)^{-1} F^{\prime}(x)-I\right)\left(x^{\star}-x\right)+\mathcal{B}(x)^{-1} r .
\end{aligned}
$$

We shall find upper bounds on the composers of (2.19). Using $(2.2)-(2.4)$, and (2.17), we have in turn:

$$
\begin{aligned}
& \|-F^{\prime}(x)^{-1}\left(F\left(x^{\star}\right)-\left(F(x)+F^{\prime}(x)\left(x^{\star}-x\right)\right)+\left(x^{\star}-x\right) \|\right. \\
& \leq\left\|F^{\prime}(x)^{-1} F^{\prime}\left(x^{\star}\right)\right\|\left\|F^{\prime}\left(x^{\star}\right)^{-1}\left(F\left(x^{\star}\right)-F(x)-F^{\prime}(x)\left(x^{\star}-x\right)\right)\right\|+\left\|x^{\star}-x\right\| \\
& \leq\left\|F^{\prime}(x)^{-1} F^{\prime}\left(x^{\star}\right)\right\| \int_{0}^{1}\left\|F^{\prime}\left(x^{\star}\right)^{-1}\left(F^{\prime}\left(x+\tau\left(x^{\star}-x\right)\right)-F^{\prime}(x)\right)\right\| d \tau\left\|x^{\star}-x\right\|+ \\
& \quad\left\|x^{\star}-x\right\| \\
& \leq \frac{\int_{0}^{1}\left(f^{\prime}\left(\left\|x-x^{\star}\right\|\right)-f^{\prime}\left(\tau\left\|x-x^{\star}\right\|\right)\right) d \tau\left\|x-x^{\star}\right\|}{\left|f_{0}^{\prime}\left(\left\|x-x^{\star}\right\|\right)\right|}+\left\|x^{\star}-x\right\| \\
& \leq \frac{f(0)-f\left(\left\|x-x^{\star}\right\|\right)+f^{\prime}\left(\left\|x-x^{\star}\right\|\right)\left\|x-x^{\star}\right\|}{-f_{0}^{\prime}\left(\left\|x-x^{\star}\right\|\right)}+\left\|x-x^{\star}\right\| \\
& =g\left(\left\|x-x^{\star}\right\|\right)+\left\|x-x^{\star}\right\|,
\end{aligned}
$$


where,

$$
g(t)=\frac{-f(t)+f^{\prime}(t) t}{-f_{0}^{\prime}(t)}
$$

We also have:

$\left\|F^{\prime}(x)^{-1} \mathcal{P}^{-1}\right\|\|\mathcal{P} F(x)\| \leq\left\|\left(\mathcal{P}^{\prime} F(x)\right)^{-1}\right\|\left\|\mathcal{P} F^{\prime}(x)\right\|\left(g\left(\left\|x-x^{\star}\right\|\right)+\left\|x-x^{\star}\right\|\right)$.

Using 2.10, 2.11, 2.15 2.19), and 2.20), we have in turn:

$$
\begin{aligned}
\left\|x_{+}-x^{\star}\right\| \leq & \left\|\mathcal{B}(x)^{-1} F^{\prime}(x)\right\|\left\|F^{\prime}(x)^{-1} F^{\prime}\left(x^{\star}\right)\right\| \times \\
& \left\|F^{\prime}\left(x^{\star}\right)^{-1}\left(F\left(x^{\star}\right)-\left(F(x)+F^{\prime}(x)\left(x^{\star}-x\right)\right)\right)\right\|+ \\
& \left\|\mathcal{B}(x)^{-1} F^{\prime}(x)-I\right\|\left\|x-x^{\star}\right\|+ \\
\leq & \left\|\mathcal{B}(x)^{-1} F^{\prime}(x)\right\|\left\|F^{\prime}(x)^{-1} \mathcal{P}^{-1}\right\|\|\mathcal{P} r\| \\
& w_{1} g\left(\left\|x-x^{\star}\right\|\right)+w_{2}\left\|x-x^{\star}\right\|+ \\
& w_{1} \theta\left\|F^{\prime}(x)^{-1} \mathcal{P}^{-1}\right\|\|\mathcal{P} F(x)\| \\
\leq & w_{1} g\left(\left\|x-x^{\star}\right\|\right)+w_{2}\left\|x-x^{\star}\right\|+ \\
& w_{1} \theta\left(g\left(\left\|x-x^{\star}\right\|\right)+\left\|x-x^{\star}\right\|\right) .
\end{aligned}
$$

By letting $x_{+}=x_{n+1}, x=x_{n}$ in 2.24 , we get:

$$
\begin{aligned}
\left\|x_{n+1}-x^{\star}\right\| \leq & w_{1} g\left(\left\|x_{n}-x^{\star}\right\|\right)+w_{2}\left\|x_{n}-x^{\star}\right\|+ \\
& w_{1} \theta\left(g\left(\left\|x_{n}-x^{\star}\right\|\right)+\left\|x_{n}-x^{\star}\right\|\right) \\
\leq & \alpha_{0}\left\|x_{n}-x^{\star}\right\|<\left\|x_{n}-x^{\star}\right\|,
\end{aligned}
$$

which imply $x_{n+1} \in U\left(x^{\star}, \sigma_{0}\right)$, and $\lim _{n \longrightarrow \infty} x_{n}=x^{\star}$.

That completes the proof of Theorem 2.1.

Remark 2.2. If $f(t)=f_{0}(t)$ on $[0, R)$, then, Theorem 2.1 reduces to Theorem 4 in [9]. Note that in view of (2.3), there exists function $f_{0}$ satisfying (2.2), (2.4), and $(2.5)$.

Therefore, we have not introduced an additional hypothesis. In practice, the computation of function $f$ requires that of $f_{0}$.

Moreover,

$$
f_{0}^{\prime}(t) \leq f^{\prime}(t) \quad t \in[0, R)
$$

holds in general, and $\frac{f^{\prime}(t)}{f_{0}^{\prime}(t)}$ can be arbitrarily large [3]- 6$]$.

Denote by $\rho, \sigma, \alpha$ the constants (used in [9]) corresponding to $\rho_{0}, \sigma_{0}, \alpha_{0}$ respectively by simply replacing $f_{0}$ by $f$ in definitions $(2.8), 2.9$, and $(2.15)$.

In view of (2.25), we have:

$$
\begin{gathered}
\rho \leq \rho_{0} \\
\sigma \leq \sigma_{0} \\
\alpha_{0} \leq \alpha
\end{gathered}
$$


If strict inequality holds in (2.25), then so does in $(2.28)$. Hence, the estimates on the distances have been improved.

Let us examine some special choices of functions $f_{0}$, and $f$.

Case 1: (Lipschitz case)

$$
\begin{gathered}
f_{0}(t)=\frac{K_{0} t^{2}}{2}-t, \quad f(t)=\frac{K t^{2}}{2}-t, \\
\mathcal{B}\left(x_{n}\right)=F^{\prime}\left(x_{n}\right), \quad \mathcal{P}_{n}=I, \quad w_{1}=1, \quad \text { and } w_{2}=0 \quad(n \geq 0) .
\end{gathered}
$$

Then, we get:

$$
\begin{gathered}
\alpha_{0}=(1+\theta) \frac{K\left\|x-x^{\star}\right\|}{2\left(1-K_{0}\left\|x_{0}-x^{\star}\right\|\right)}+\theta, \\
\alpha=(1+\theta) \frac{K\left\|x-x^{\star}\right\|}{2\left(1-K\left\|x_{0}-x^{\star}\right\|\right)}+\theta,
\end{gathered}
$$

which imply

$$
\sigma_{0}=\min \left\{K, \frac{2(1-\theta)}{2(1-\theta) K_{0}+(1+\theta) K}\right\},
$$

and

$$
\sigma=\min \left\{K, \frac{2(1-\theta)}{(3-\theta) K}\right\}
$$

If $\theta=0$, we obtain:

$$
r_{0}=\min \left\{K, \frac{2}{2 K_{0}+K}\right\},
$$

and

$$
r=\min \left\{K, \frac{2}{3 K}\right\}
$$

which imply

$$
r \leq r_{0}
$$

Estimate 2.35 shows that we obtain a larger radius of convergence than in [9]. Below, we provide a numerical example where $r<r_{0}$ (see Example 2.3 .

Case 2: (Smale-Wang case)

$$
f_{0}(t)=\frac{t}{1-\gamma_{0} t}-2 t, \quad f(t)=\frac{t}{1-\gamma t}-2 t \quad\left(\gamma \leq \gamma_{0}\right)
$$

and choose:

$$
R=\frac{1}{\gamma_{0}}
$$

The rest is left to the motivated reader.

More choices of functions $f_{0}$, and $f$ can be found in [9]. 
Example 2.3. Let $\mathcal{X}=\mathcal{Y}=(-\infty,+\infty), \mathcal{D}=U\left(x^{\star}, R\right)=U(0,1)$, and define function $F$ on $\mathcal{D}$ by:

$$
F(x)=e^{x}-1
$$

Then we obtain:

$$
K_{0}=e-1, \quad K=e .
$$

In view (2.33), and (2.34), we get:

$$
r=\frac{2}{3 K}=.245252961<r_{0}=\frac{2}{2 K_{0}+K}=.324947231 .
$$

\section{REFERENCES}

1. I.K. Argyros, Relation between forcing sequences and inexact Newton iterates in Banach space, Computing. 63 (1999), 134-144. 1

2. I.K. Argyros, Forcing sequences and inexact Newton iterates in Banach space, Appl. Math. Lett. 13 (2000), 69-75.

3. I.K. Argyros, A unifying local-semilocal convergence analysis and applications for twopoint Newton-like methods in Banach space, J. Math. Anal. Appl. 298 (2004), 374-397. 1. 2.2

4. I.K. Argyros, Computational theory of iterative methods, Series: Studies in Computational Mathematics, 15, Editors: C.K. Chui and L. Wuytack, Elsevier Publ. Co., New York, USA, 2007. 1, 2

5. I.K. Argyros, Convergence and applications of Newton-type iterations, Springer-Verlag Publ., New York, 2008. 1, 2

6. I.K. Argyros, On the semilocal convergence of inexact Newton methods in Banach spaces, J. Comput. Appl. Math. in press, doi:10.1016/j.cam.2008.10.005. 2.2

7. J. Chen, W. Li, Convergence behaviour of inexact Newton methods under weak Lipschitz condition, J. Comput. Appl. Math. 191 (2006), 143-164. 1

8. J.F. Dennis, Toward a unified convergence theory for Newton-like methods, in Nonlinear Functional Analysis and Applications (L.B. Rall, ed.), Academic Press, New York, (1971), $425-472$.

9. O.P. Ferreira, M.L.N. Goncalves, Local convergence analysis of inexact Newton-like methods under majorant condition, preprint, http://arxiv.org/abs/0807.3903?context=math.OC. (document), 1, 2.2, 2.2, 2.2

10. X. Guo, On semilocal convergence of inexact Newton methods, J. Comput. Math. 25 (2007), $231-242$.

11. Z.A. Huang, Convergence of inexact Newton method, J. Zhejiang Univ. Sci. Ed. 30 (2003), 393-396.

12. L.V. Kantorovich, G.P. Akilov, Functional Analysis, Pergamon Press, Oxford, 1982.2

13. B. Morini, Convergence behaviour of inexact Newton methods, Math. Comp. 68 (1999), 1605-1613.

14. F.A. Potra, Sharp error bounds for a class of Newton-like methods, Libertas Mathematica. 5 (1985), 71-84.

15. X.H. Wang, C. Li, Convergence of Newton's method and uniqueness of the solution of equations in Banach spaces, II, Acta Math. Sin. (Engl. Ser.) 19 (2003), 405-412. 1.

1 Cameron university, Department of Mathematics Sciences, Lawton, OK 73505, USA.

E-mail address: iargyros@cameron.edu 
2 Poitiers university, Laboratoire de Mathématiques et Applications, Bd. Pierre et Marie Curie, Téléport 2, B.P. 30179, 86962 Futuroscope Chasseneuil Cedex, France.

E-mail address: said.hilout@math.univ-poitiers.fr 